\title{
Suppression of the dynamic response of cracked beams with active control
}

\author{
Meng-Ju Lin ${ }^{1, a}$, Yu-Cheng Chen ${ }^{2}$ and Lin-Tsang Lee ${ }^{2}$ \\ ${ }^{1}$ Department of Applied Mathematics, Tunghai University.No.1727, Sec.4, Taiwan Boulevard, Taichung, Taiwan \\ ${ }^{2}$ Department of Applied Mathematics, National Chung-Hsing University, Taichung, Taiwan
}

\begin{abstract}
Recently, studies on the dynamic responses of cracked beams are quite worthy of being conferred. The theoretical dynamic properties of cracked beams previously obtained by La-DQM method were utilized to evaluate the maximum amplitudes of cracked beams. A comparatively new concept which utilized the controller gain to suppress the transverse vibration response of cracked beams in the perspective of proportional control scheme was proposed. A simulation study which applied an active control strategy to suppress the amplitude of vibration on the cracked beams with MATLAB SIMULINK software has been successfully accomplished. The maximum dynamic response of cracked beams is found to be inversely proportional to controller gain. Increasing the controller gain properly to control the vibration of the cracked beams is suggested in this study.
\end{abstract}

\section{Introduction}

Mechanic vibration has been widely applied in various fields of the industry such as: vibration analysis of vehicles, engine vibration and noise analysis, safety control of bridge vibration, stability of the micro flexible mechanical system, etc. It has become an important subject to study the damage to the beam in order to understand the integrity of beam structure that will result in the risk of structural collapse. In recent years there have been a lot of scholars observing dynamic behaviors of beam based on vibration mode, proposing theories for solving the vibration problem of cracked beam by predicting the location and size of cracks based on the natural frequency. In summary, the study on the vibration behavior of cracked beam is a subject worthy of investigation. A mathematical model including interaction between solids and fluids was proposed by Dimarogonas et al. [1]. Besides, a variety of methods were proposed to deal with the crack problems of structures. The general stiffness matrix of Euler-Bernoulli is applied on the local flexibility of beam. The linear spring models have been successfully applied in transverse cracks beams with conditions to simulate cracks by Mohammad [2]. Rizos et al. [3] employed the measured vibration modes to identify the crack locations, depths, and vibration frequencies in order to obtain the dynamic response of cracked beams. Narkis [4] employed the variation of the first two natural frequencies to obtain the locations of the cracks in the simply supported beam. The cracks are simulated as rotational springs and the beam is divided into two sections, while the proposed theoretical method is verified by finite element method. Lin et al. [5] proposed a theoretic model to describe the non-linear, three-dimensional response of a

\footnotetext{
${ }^{a}$ Corresponding author : vbenz2006@thu.edu.tw
} 
suspended cable supporting an array of discrete masses. It is applied to obtain the eigenvalues of that complicated system, and the transfer matrix of this method can be applied to the free vibration of complex mass system. Lin et al [6] employed the transfer matrix to obtain the natural frequencies of any number of cracks. This is an easy and effective method to obtain the solution of vibration mode, which will result in the numeric results very similar to the experimental results. Later on, Wang et al. [7] proposed simplified sectional flexibility factor to simulate the study on single open crack, where the stress intensity factor is simplified, and the transfer matrix is used to establish the eigenvalue equation. It provided an easy and effective way for analyzing cracked beam. Bellman et al. [8] was the first to propose DQM in 1971 to approximate the integral item to be solved by the "quadrature" approach while approximating the first order differentiation by extending the sum of linear function. Lee et al. [9] applied local adaptive differential quadrature method (La-DQM) to the study of identification of beams with multiple cracks which led to fairly good results.

As an important public facility, bridges provide people the traffic convenience at the cost of the problems of vibration and noise. Experts have developed a variety of methods and theories to avoid the negative effects of vibrations. Among them, the passive control methods and the active control methods are the commonest [10]. The passive control is mainly achieved by changing the property parameters, mass, damping and strength of the system itself. And the active control utilizes the vibration signals of the sensor and counteracts the vibration by the control force generated according to the results of the calculation of the controller [11]. Several scholars utilized discretization of finite difference to conduct the active control of cantilever beam and simulate the dynamic state of structure of the beams through the discretization process. After this, they employed active control to do the control [12-14].

An active control model was proposed by Tavakolpour et al. [15] to suppress the dynamic response of cracked beams. The methodology for the active vibration control of a flexible plate structure employed by Tavakolpour et al. is known as high gain feedback (HGF) regulator. Proportional control scheme is then applied to analyze the final response on the maximum amplitude of cracked beam which can be used to minimize the negative effect of vibration. The amplitude of cracked beam is reduced by increasing the controller gain. This study is to provide a suitable controller gain for proportional control scheme to suppress the dynamic response of cracked beams. The control performance is evaluated by varying the proportional gain until it reaches the optimum value where attenuation percentage of the maximum amplitudes of the cracked beam is obtained.

\section{Transverse vibration response of a cracked beam}

\subsection{Calculation of eigenvalues}

Considering an Euler Bernoulli beam with length $\mathrm{L}$ with a crack located at $X_{1}$ thus separating this beam into $L_{1}, L_{2}$ as shown in Figure 1. In accordance with literature [2], the governing equation for every section of separated beam can be shown as:

$$
E I \frac{\partial^{4} Y_{i}(X, T)}{\partial X^{4}}+\rho A \frac{\partial^{2} Y_{i}(X, T)}{\partial T^{2}}=0, X_{i-1}<X<X_{i}, i=1,2
$$

where $E$ is the modulus of elasticity , $I$ is the moment of inertia of the Euler Bernoulli beam crosssection, $\rho$ is the density of material and $A$ is the cross-section area. 


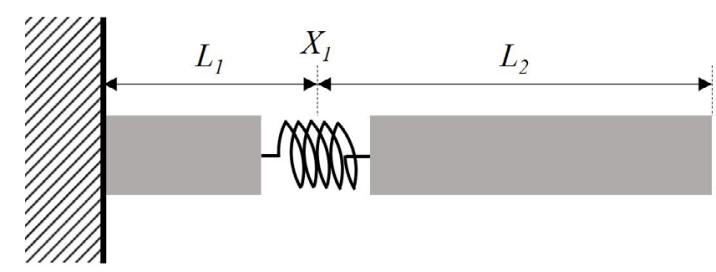

Figure 1. Schematic diagram of a cantilever beam with one crack.

The following quantities are introduced for non-dimensional analysis:

$$
y=\frac{Y}{L}, x=\frac{X}{L}, t=T, x_{i}=\frac{X_{i}}{L}, y_{i}=\frac{Y_{i}}{L}, l_{i}=\frac{L_{i}}{L} .
$$

The external force $f(x, t)$ is applied to the right free end of the cantilever beam, the governing equation for every section of separated beam can be denoted in the non-dimensional form as shown below:

$$
\frac{E I}{L^{4}} \frac{\partial^{4} y_{i}(x, t)}{\partial x^{4}}+\rho A \frac{\partial^{2} y_{i}(x, t)}{\partial t^{2}}=f(x, t), \quad x_{i-1}<x<x_{i}, \quad i=1,2
$$

By applying separation of variables $y_{i}(x, t)=w_{i}(x) e^{j \omega t}$, and letting the external force $f(x, t)=0$ then the governing equation of (3) will lead to

$$
\frac{E I}{L^{4}} w_{i}^{(I V)}(x)-\rho A \omega^{2} w_{i}(x)=0,
$$

and the differential equation for free vibration can be written as

$$
w_{i}^{(I V)}(x)-\lambda^{4} w_{i}(x)=0, x_{i-1}<x<x_{i}, \quad i=1,2
$$

where $\lambda^{4}=\frac{\rho A \omega^{2} L^{4}}{E I}$ is eigenvalue, and $\omega$ is the natural frequency, and the compatibility conditions of the locations of cracks are as shown below:

$$
\begin{gathered}
w_{1}\left(x_{1}^{-}\right)=w_{2}\left(x_{1}^{+}\right), \\
w_{1}^{\prime \prime}\left(x_{1}^{-}\right)=w_{2}^{\prime \prime}\left(x_{1}^{+}\right), \\
w_{1}^{\prime \prime \prime}\left(x_{1}^{-}\right)=w_{2}^{\prime \prime \prime}\left(x_{1}^{+}\right), \\
w_{2}^{\prime}\left(x_{1}^{+}\right)-w_{1}^{\prime}\left(x_{1}^{-}\right)=c^{*} w_{2}^{\prime \prime}\left(x_{1}^{+}\right) .
\end{gathered}
$$

Where $c^{*}$ is the non-dimensional crack section flexibility coefficient as

$$
c=\frac{E I c}{L}=\frac{2 \pi\left(1-v^{2}\right)\left[1-\left(1-\frac{a}{h}\right)^{2}\right]}{9\left(1-\frac{a}{h}\right)^{2}} \frac{h}{L},
$$


and $c=\frac{2 \pi\left(1-v^{2}\right)\left[1-\left(1-\frac{a}{h}\right)^{2}\right] h}{9 E I\left(1-\frac{a}{h}\right)^{2}}$ is the section flexibility coefficient, $a$ refers to the depth of the crack.

Every section of beam can be connected by employing transfer matrix method [5] for calculation of eigenvalue of the entire system. The general solution of equation (5), for each segment is as shown below:

$$
\begin{gathered}
w_{i}(x)=A_{i} \sin \left[\lambda\left(x-x_{i-1}\right)\right]+B_{i} \cos \left[\lambda\left(x-x_{i-1}\right)\right]+C_{i} \sinh \left[\lambda\left(x-x_{i-1}\right)\right]+D_{i} \cosh \left[\lambda\left(x-x_{i-1}\right)\right] \\
x_{i-1}<x<x_{i}, \quad i=1,2 .
\end{gathered}
$$

Where $A_{i}, B_{i}, C_{i}$ and $D_{i}$ are constants. These constants in the (i+1) $)^{\text {th }}$ segment $\left(A_{i+1}, B_{i+1}, C_{i+1}\right.$ and $\left.D_{i+1}\right)$ are related to those in the $\mathrm{i}^{\text {th }}$ segment $\left(A_{i}, B_{i}, C_{i}\right.$ and $\left.D_{i}\right)$ through compatibility conditions in equation (6). The eigenvalue can be obtained in accordance with the transfer matrix of literature [6].

\subsection{Generalized coordinates}

Based on the reason, $g_{r}(t) \approx 0$ for $r \geq 6$, so the transverse vibration response of a cracked beam can be shown as:

$$
y_{i}(x, t)=\sum_{r=1}^{5} w_{r i}(x) g_{r}(t), \quad i=1,2
$$

Substituting equation (9) into equation (3), the generalized coordinates function $g_{r}(t)$ of cracked beam can be expressed via orthogonality and normalization as:

$$
\omega_{r}^{2} g_{r}(t)+\frac{d^{2} g_{r}(t)}{d t^{2}}=n_{r}(t)
$$

where $n_{r}(t)=\int_{0}^{l_{1}} w_{r 1}(x) f(x, t) d x+\int_{l_{1}}^{1} w_{r 2}(x) f(x, t) d x$

The initial conditions of Euler Bernoulli beam are:

$$
g_{r}(0)=0, \frac{d g_{r}(0)}{d t}=0
$$

Based on the model with the initial conditions (11), and performing Laplace transform to equations (10) and (11), the equation (10) becomes:

$$
\omega_{r}^{2} g_{r}(s)+s^{2} g_{r}(s)=N_{r}(s) .
$$

The transfer function of the flexible beam system can be obtained from equation (12) as:

$$
G_{r}(s)=\frac{g_{r}(s)}{N_{r}(s)}=\frac{1}{\omega_{r}^{2}+s^{2}} .
$$




\section{Proportional control scheme}

Experts and scholars have developed numerous methods and theories to avoid the negative impacts of vibration. The proportional control scheme is considered in this study as the scheme for controlling the vibration of flexible beam system. When an external force is applied to the beam, a counter force will be generated by the actuator to compensate the displacement caused by the external force. This dynamical system attempts to reduce the amplitude of cracked beam. The closed-loop active vibration control system is as shown in Figure. 2. In the proportional controller, the control action is proportional to the error, and we can represent the controller as a gain, $K$. The transfer function of the flexible beam system will be plant, $G$. The sensor, $G_{s}$, measures the output of the system we are controlling. The error expression tells us how much the output deviates from the input. The device which performs the subtraction to compute the error, $e$, is a comparator. The controller will generally try to reduce the error of displacement between the reference value and its actual value until we get the minimum error. The effectiveness of the controller action depends on the optimum setting of its proportional gain.

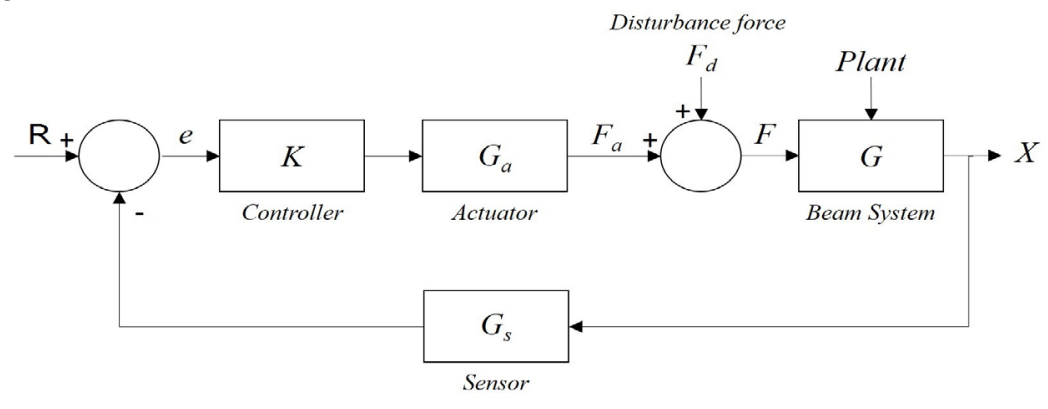

Figure 2 . Proportional Control Scheme.

For the closed-loop system in Fig. 2, the reference input $\mathrm{R}$ is set to zero. The input to the actuator $G_{a}$ is $-G_{s} K X$, the output is $F_{a}=-G_{a} G_{s} K X$, and $F_{a}, F_{d}$ of the flexible beam system are actuating force and disturbing force respectively. Thus, the total external force fed into the flexible beam system is given by:

$$
F=F_{a}+F_{d}=-G_{a} G_{s} K X+F_{d}
$$

Thus the displacement $X$ of the flexible beam can be shown as:

$$
X=G F_{d}\left\{\frac{1}{1+G_{a} G_{s} K G}\right\}
$$

The beam displacement can be reduced by adjusting the gain of controller $K$ in accordance with equation (15), and the less displacement indicates less beam vibration. Equation (15) indicates that the $X$ value is inversely proportional to controller gain, $K$.

The entire control system can be simplified based on the unity of actuator and sensor. The equation after simplification can be shown as:

$$
X=G F_{d}\left\{\frac{1}{1+K G}\right\}
$$

Based on equation (16), the displacement of the flexible beam is reduced by increasing the controller gain, $K$. 


\section{Numerical analysis and discussions(simulation results)}

The proposed methods are used to solve the beam vibration problems with un-cracked and cracked beams. In order to validate the method in this article, the numerical results are compared with the available data. First is the case of the un-cracked cantilever beam shown in Figure 3 with length $L=0.3 m$, height $h=0.02 m$, width $b=0.02 m$, area $A=0.02 \times 0.02 m^{2}$, Young's modulus $E=2.06 \times 10^{11} \mathrm{~N} / \mathrm{m}^{2}$, and mass density $\rho=7800 \mathrm{~kg} / \mathrm{m}^{3}$. The external force $f=10 \sin \left(\omega_{f} t\right), \omega_{f}=2 \pi \neq \omega_{r}$ and $r=1,2,3, \cdots \cdots$, is applied to the right free end of the beam. The natural frequencies of an un-cracked cantilever beam obtained via Theoretical method and La-DQM are as shown in Table 1. With proportion control scheme in this article, the percentage of the maximum vibration amplitudes of un-cracked beam can be reduced by adjusting higher controller gain, $K$ value. The percentage reductions of the maximum vibration amplitudes before and after the control over the beam with different proportional gains are obtained as shown in Table 2.

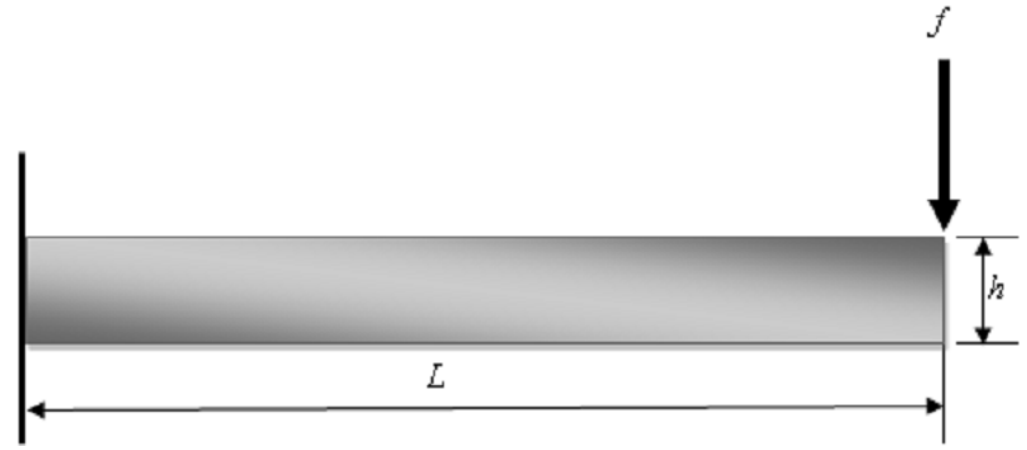

Figure 3. Illustration of an un-cracked cantilever beam.

Table 1. The natural frequencies of an un-cracked cantilever beam

\begin{tabular}{|c|c|c|c|}
\hline Method & First frequency $(\mathrm{Hz})$ & Second frequency $(\mathrm{Hz})$ & Third frequency $(\mathrm{Hz})$ \\
\hline $\begin{array}{c}\text { Theoretical method } \\
\text { by this article }\end{array}$ & 184.45 & 1156.12 & 3237.19 \\
\hline La-DQM & 184.48 & 1156.12 & 3237.08 \\
\hline
\end{tabular}

Table 2. The percentage reductions of maximum vibrations before and after the control over the un-cracked cantilever beam.

\begin{tabular}{|c|c|c|c|c|}
\hline$K$ value & $\begin{array}{c}\text { Maximum vibration } \\
\text { before the control }\end{array}$ & $\begin{array}{c}\text { Maximum vibration } \\
\text { after the control }\end{array}$ & $\begin{array}{c}\text { Percentage } \\
\text { reduction }(\%)\end{array}$ & $\begin{array}{c}\text { rate of reduction per unit gain (\%) } \\
\text { (Percentage reduction/ } K \text { value) }\end{array}$ \\
\hline 10,000 & $3.9844 \mathrm{E}-06$ & $3.9587 \mathrm{E}-06$ & 0.645016 & $\begin{array}{c}6.45016 \mathrm{E}-05 \\
(0.645016 / 10000)\end{array}$ \\
\hline 20,000 & $3.9844 \mathrm{E}-06$ & $3.9225 \mathrm{E}-06$ & 1.553559 & $7.76779 \mathrm{E}-05$ \\
\hline 50,000 & $3.9844 \mathrm{E}-06$ & $3.8175 \mathrm{E}-06$ & 4.188836 & $8.37767 \mathrm{E}-05$ \\
\hline 100,000 & $3.9844 \mathrm{E}-06$ & $3.6530 \mathrm{E}-06$ & 8.317438 & $8.31744 \mathrm{E}-05$ \\
\hline 500,000 & $3.9844 \mathrm{E}-06$ & $2.8339 \mathrm{E}-06$ & 28.875113 & $5.77502 \mathrm{E}-05$ \\
\hline $5,000,000$ & $3.9844 \mathrm{E}-06$ & $7.5218 \mathrm{E}-07$ & 81.121875 & $1.62244 \mathrm{E}-05$ \\
\hline
\end{tabular}

Next, the cantilever beam with a single crack is chosen as shown in Figure 4 with length $L=0.3 m$, height $h=0.02 m$, width $b=0.02 m$, area $A=0.02 \times 0.02 \mathrm{~m}^{2}$, Young's modulus $E=2.06 \times 10^{11} \mathrm{~N} / \mathrm{m}^{2}$, and mass density $\rho=7800 \mathrm{~kg} / \mathrm{m}^{3}$. The crack is located at $X_{1}=0.14 \mathrm{~m}$ from the fixed end with the depth $a_{1}=0.01 \mathrm{~m}$. The crack depth ratio is $a / h=0.01 / 0.02=0.5$ and the 
crack location $X_{1} / L=0.14 / 0.3=0.4667$. The external force $f=10 \sin \left(\omega_{f} t\right), \omega_{f}=2 \pi \neq \omega_{r}$ and $r=1,2,3, \cdots \cdots$, is applied to the right free end of the cantilever beam. The natural frequencies of the cracked beam as shown in Table 3 are obtained via Numerical results, Experiment results, Theoretical results and results of La-DQM. With the proportion control scheme in this article, the percentage reductions of maximum vibration amplitudes before and after the controller with different proportional gains are obtained. The percentage reductions of maximum vibrations before and after the control over the beam based on different $K$ values are as shown in Table 4.

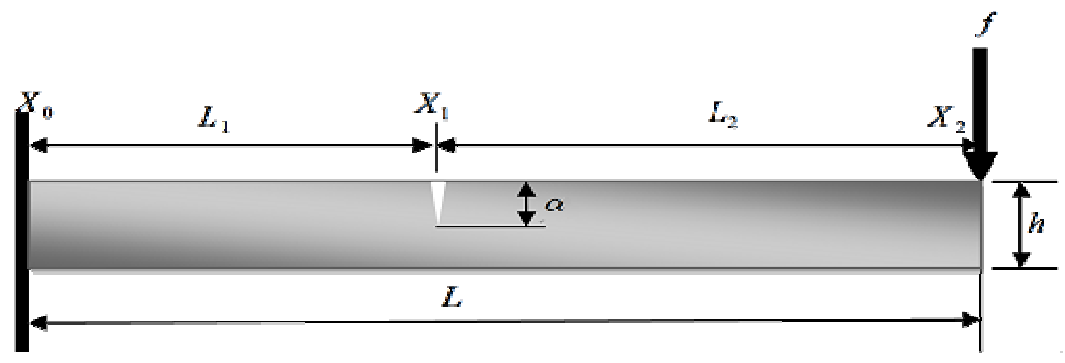

Figure 4. Illustration of a cracked cantilever beam.

Table 3. The natural frequencies of a cracked cantilever beam.

\begin{tabular}{|c|c|c|c|}
\hline Method & First frequency $(\mathrm{Hz})$ & Second frequency(Hz) & Third frequency(Hz) \\
\hline Numerical results from[6] & 173.88 & 988.45 & 3211 \\
\hline Experiment results from[3] & 171 & 987 & 3034 \\
\hline $\begin{array}{c}\text { Theoretical method [5] } \\
\text { by this article }\end{array}$ & 178.06 & 1043.74 & 3219.98 \\
\hline La-DQM[8] & 178.06 & 1043.74 & 3219.99 \\
\hline
\end{tabular}

Table 4. The percentage maximum vibrations before and after the control over the cracked cantilever beam.

\begin{tabular}{|c|c|c|c|c|}
\hline$K$ value & $\begin{array}{c}\text { Maximum vibration } \\
\text { before the control }\end{array}$ & $\begin{array}{c}\text { Maximum vibration } \\
\text { after the control }\end{array}$ & $\begin{array}{c}\text { Percentage } \\
\text { reduction }(\%)\end{array}$ & $\begin{array}{c}\text { rate of reduction per unit gain (\%) } \\
\text { (Percentage reduction/ } K \text { value) }\end{array}$ \\
\hline 10000 & $3.9844 \mathrm{E}-06$ & $3.9587 \mathrm{E}-06$ & 0.645016 & $\begin{array}{c}6.45016 \mathrm{E}-05 \\
(0.645016 / 10000)\end{array}$ \\
\hline 20000 & $3.9844 \mathrm{E}-06$ & $3.9225 \mathrm{E}-06$ & 1.553559 & $7.76779 \mathrm{E}-05$ \\
\hline 50000 & $3.9844 \mathrm{E}-06$ & $3.8175 \mathrm{E}-06$ & 4.188836 & $8.37767 \mathrm{E}-05$ \\
\hline 100000 & $3.9844 \mathrm{E}-06$ & $3.6530 \mathrm{E}-06$ & 8.317438 & $8.31744 \mathrm{E}-05$ \\
\hline 500000 & $3.9844 \mathrm{E}-06$ & $2.8339 \mathrm{E}-06$ & 28.875113 & $5.77502 \mathrm{E}-05$ \\
\hline 5000000 & $3.9844 \mathrm{E}-06$ & $7.5218 \mathrm{E}-07$ & 81.121875 & $1.62244 \mathrm{E}-05$ \\
\hline
\end{tabular}

This study is to obtain transverse vibration responses via natural frequencies of un-cracked and cracked beams obtained in accordance with aforementioned conditions of beams, and then the proportion control scheme will be applied to carry out beam control based on different $K$ values of controller with $K=10000 、 20000 、 50000,100000,500000$ and 5000000. The percentage reductions of the maximum amplitude before and after the control are obtained for the comparison between the transverse vibration response relationship diagrams of cracked and un-cracked beams before and after the control. The results reveal that proportional control scheme has effectively reduced the vibration amplitudes of un-cracked beam and cracked beam. The higher the proportional gain results into the better attenuation percentage of the maximum vibration amplitudes of un-cracked beam and cracked beam. The comparisons between the transverse vibration response diagrams of un-cracked and cracked beams before and after the control with different proportional gains are as shown in Figure 5 
to Figure 10. The black solid line and black dotted line in the figure represent the transverse vibration responses of the uncontrolled and controlled un-cracked beams respectively. The red solid line and red dotted line in the figure represent the transverse vibration responses of the uncontrolled and controlled cracked beams respectively.

Proportional control scheme has been proven its capability of reducing the maximum vibration amplitude of un-cracked beam and cracked beam. The performance of the controller in suppressing the maximum vibration amplitudes of beams depends on the proportional gain. That is to say, increasing the proportional gain will effectively reduce the maximum amplitude of the system. It is noted that, although the higher the proportional gain gets the better attenuation percentage of the maximum vibration amplitudes of the cracked beam but it will lead to higher costs and higher actuator force. Based on these reasons, the highest suitable value of the proportional gain obtained is 100000 , as shown in Table 2 and Table 4, which will effectively restrain the maximum vibration amplitude of un-cracked beam and cracked beam with the percentages at $6.498656 \%$ and $8.317438 \%$ respectively. It has the more effective decline the vibration amplitudes and the better rate of decline per unit proportional gain.

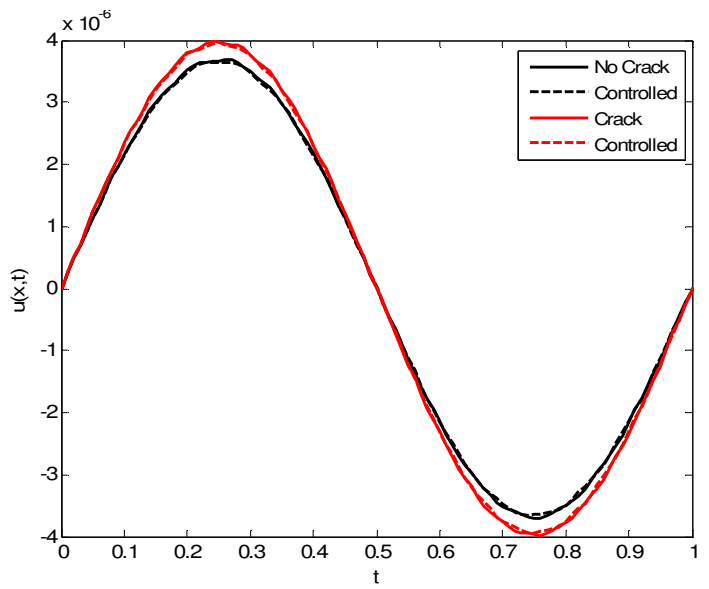

Figure 5. The transverse vibration response diagram of un-cracked and cracked beams before and after control with $K=10000$.

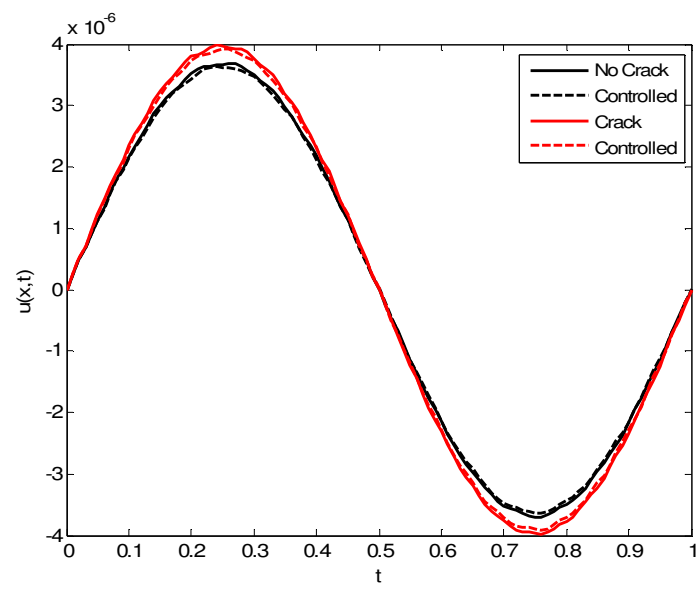

Figure 6. The transverse vibration response diagram of un-cracked and cracked beams before and after control with $K=20000$. 


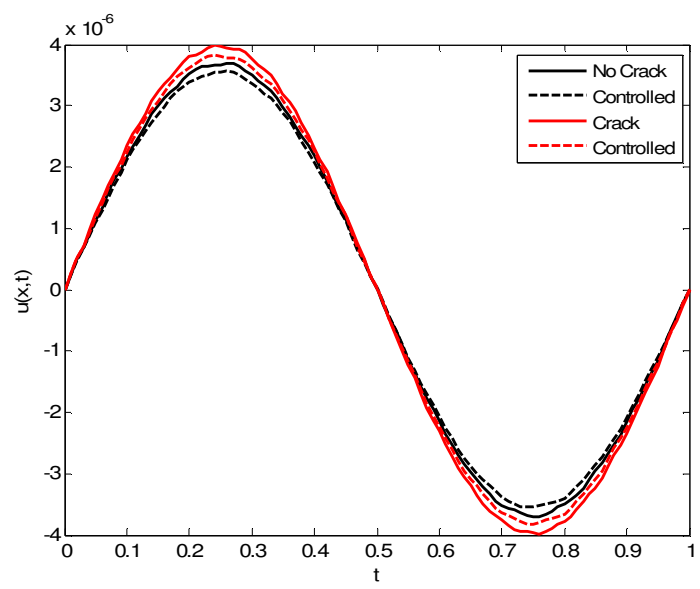

Figure 7. The transverse vibration response diagram of un-cracked and cracked beams before and after control with $K=50000$.

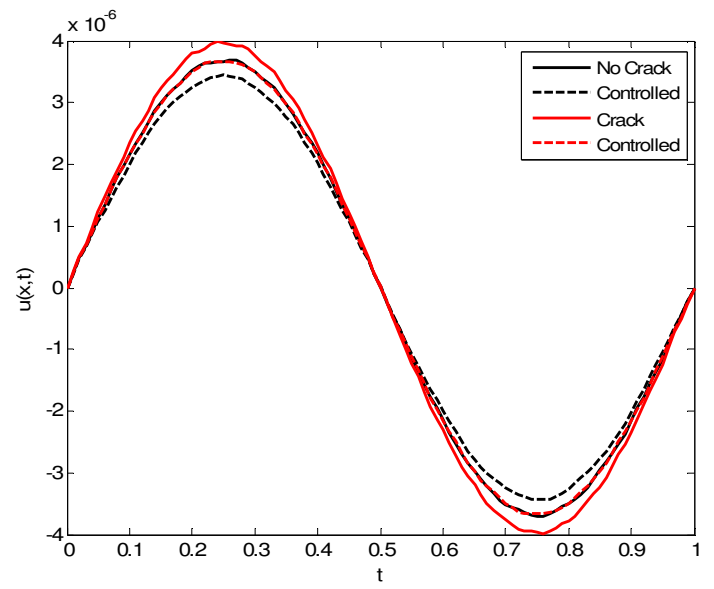

Figure 8. The transverse vibration response diagram of un-cracked and cracked beams before and after control with $K=100000$.

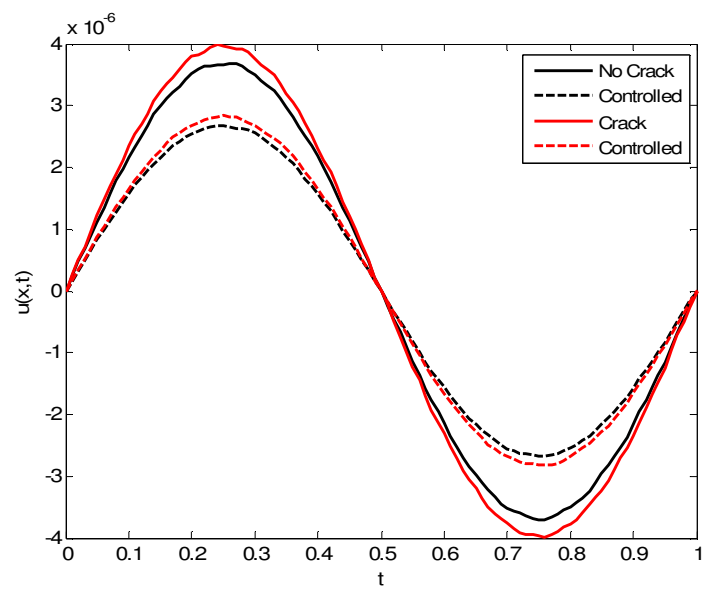

Figure 9. The transverse vibration response diagram of un-cracked and cracked beams before and after control with $K=500000$. 


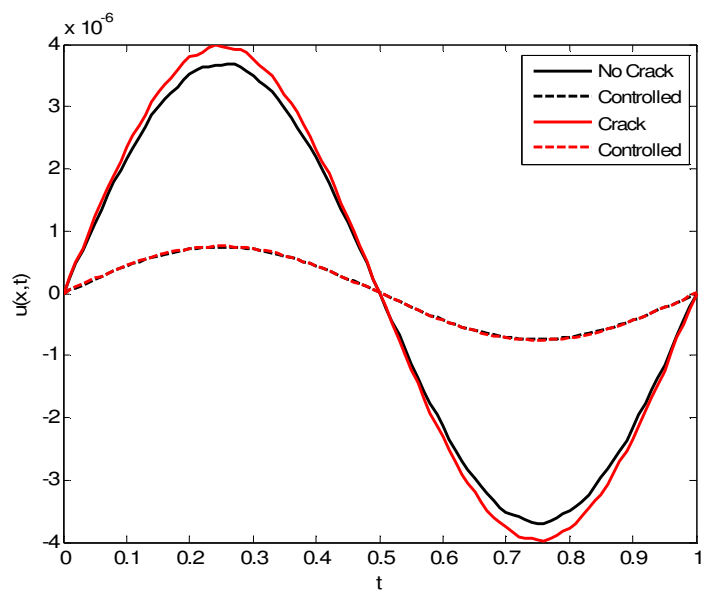

Figure 10. The transverse vibration response diagram of un-cracked and cracked beams before and after control with $K=5000000$.

\section{Conclusions}

A simulation study has been successfully accomplished by applying an active control strategy to suppress the amplitude of vibration on the cracked beam with MATLAB SIMULINK software. This simulation model of cracked beams which was represented through the operation of massless rotational spring and the two beams are connected by the compatibility equations. It was demonstrated that the proposed active controller effectively controlled transverse vibration response of the flexible cracked beam system. From the results, it reveals that proportional control scheme has significantly reduced the maximum vibration amplitude of cracked beams. However, the controller gain needs to be selected appropriately because if the proportional gain is increased beyond some values it may result in higher costs and higher actuator force. From Table 4, it is seen that the highest suitable value of the proportional gain obtained is 100000 . It can effectively suppress the maximum vibration amplitude percentage of cracked beam with the percentage of $8.317438 \%$ while maintaining better rate of reduction for per unit proportional gain value. Simulation results demonstrate that the proposed scheme is applicable effectively to vibration of cracked beam. According to the result of this research, active control system can be applied successfully to the suppression of dynamic response of cracked beam. This study offers a promising approach to realize control system in real time application.

\section{References}

1. A. D. Dimarogonas and S. A. Paipetis, Analytical methods in rotor dynamics (Applied Science, N. Y., USA, 1983)

2. M. H. Dado, Appl. Acoust., 51, 381 (1997)

3. P. F. Rizos, N. Aspragathos, and A. D. Dimarogonas, J. Sound. Vib., 138, 381 (1990)

4. Y. Narkis., J. Sound Vibr., 172, 549 (1997)

5. H. P. Lin and N. C. Perkins, J. Sound. Vib., 179, 131 (1995)

6. H. P. Lin, S. C. Chang, and J. D. Wu, J. Sound. Vib., 258, 987 (2002)

7. C. S. Wang and L. T. Lee, J. Appl. Math., 2012, 16 (2012)

8. R. B. J. Math., Anal. Appl., 34, 235 (1971)

9. T. L. Lin and J. Y. Wu, Proc. Inst. Mech. Eng. E J. Process Mech. Eng.,1 (2014)

10. A. Preumont, Vibration control of active structures: an introduction (Springer, 2011)

11. B. J. Grobbelaar, G. D. Wood, and C. P. Constancon, J. Julie., 18 (1992)

12. M. S. Saad, H. Jamaluddin, and I. Z. Mat Darus, Active vibration control of flexible beam system using proportional control scheme in finite difference simulation platform (IEEE, 2011) 
13. M. F. Hassan, M. Mailah, R. Junid, and N. A. Alang, WCE (2010)

14. A. Tavakolpour, M. Mailah, and I. Z. Mat Darus, WSTOCR, 6, 184 (2011)

15. A. R. Tavakolpour, M. Mailah, and I. Z. Mat Darus, Int. Rev. Mech. Eng., 3, 579 (2009) 